\title{
Rektorat
}

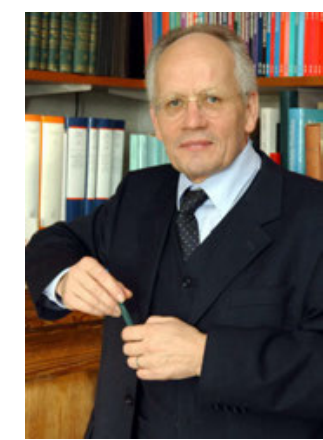

Univ.-Prof. Dr. Dr. Alfons Labisch Rektor

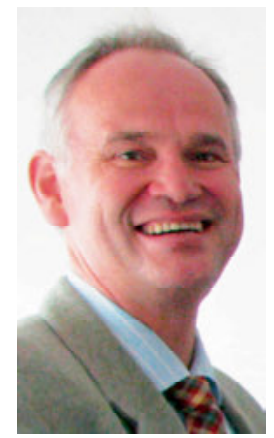

Univ.-Prof. Dr. Fritz Boege

Prorektor für Forschung, Forschungstransfer und wissenschaftlichen Nachwuchs (seit 22. November 2007)

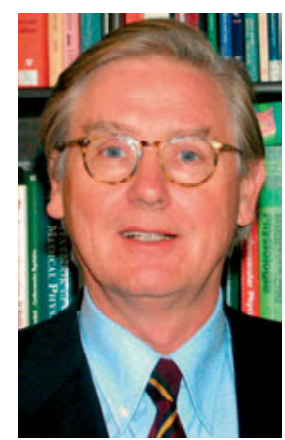

Univ.-Prof. Dr. Jürgen Schrader Prorektor für Forschung, Forschungstransfer und wissenschaftlichen Nachwuchs (bis 30. September 2007)

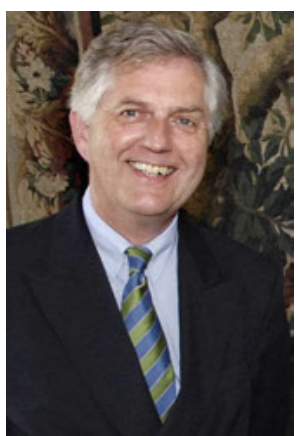

Prof. Ulf Pallme König Kanzler

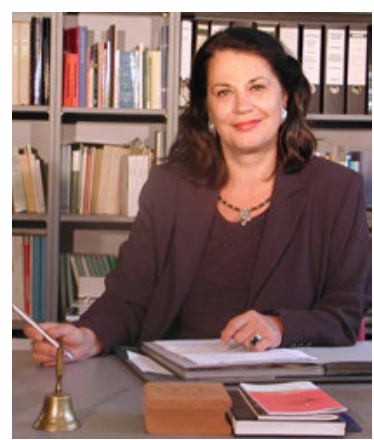

Univ.-Prof. Dr. Vittoria Borsò

Prorektorin für Internationale Angelegenheiten (bis 5. Oktober 2007)

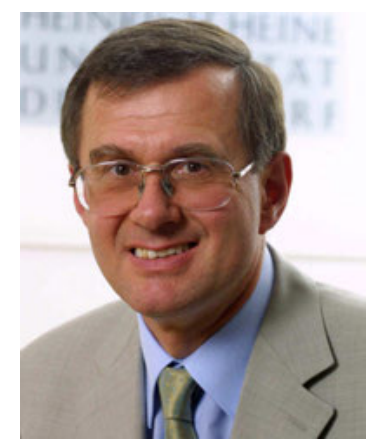

Univ.-Prof. Dr. Raimund Schirmeister Prorektor für Planung und Finanzen (bis 12. November 2007) 



\title{
Alfons Labisch
}

\section{Zur Lage und zu den Perspektiven der deutschen Universität in unserer Zeit}

\author{
Gedanken nach fünf Jahren im Amt des Rektors \\ der Heinrich-Heine-Universität Düsseldorf ${ }^{1}$
}

\section{Autonomie und Wettbewerb - Ein fundamentaler Wandel des deutschen Universitätswesens}

Seit den von Humboldt'schen Reformen zu Beginn des 19. Jahrhunderts haben sich die deutschen Universitäten nur selten so rasch und fundamental geändert, wie dies im ersten Jahrzehnt des 21. Jahrhunderts geschieht. Mit einigem Recht wäre sogar zu sagen, dass die jetzigen Reformen im deutschen Universitätswesen weit über das hinausgehen, was die Reformen Wilhelm von Humboldts jemals bedeuteten.

Dieser Aussage ließe sich durchaus einiges entgegenhalten. Im Positiven etwa der Siegeszug, mit dem sich die deutschen Universitäten seit der Mitte des 19. Jahrhunderts in einer typisch deutschen Mischung aus romantischer Welterklärung und experimentellem Empirismus zu denjenigen Stätten der Forschung wandelten, die weltweit bewundert und nachgeahmt wurden - so etwa von vielen heutigen so genannten Eliteuniversitäten der USA -, ein Universitätsmodell also, das jetzt seinem Schöpfer als Beispiel vorgehalten wird.

Im Negativen allem voran jenes säkulare Versagen, als sich die deutschen Universitäten zu Beginn der NS-Zeit ohne nennenswerten Widerstand ihrer „rassisch“ oder politisch missliebigen Kollegen entledigten, um deren Posten linientreuen Wissenschaftlern zu verschaffen. Dies führte überdies zum Verlust von durchschnittlich einem Viertel an Wissenschaftlern je Fach, darunter von 24 damals bereits gewählten beziehungsweise späteren Nobelpreisträgern.

Ebenfalls negativ war und ist jenes schleichende Vernichtungsprogramm, mit dem die Kultusminister der Länder 1977 durch den so genannten „Öffnungsbeschluss“ allen Studierwilligen einen Studienplatz versprachen, ohne im ausbrechenden Bildungsboom in notwendiger Weise in die Universitäten zu investieren. Nach diesem Sündenfall deutscher Hochschulpolitik wurden die Grundprinzipien deutscher akademischer Lehre - forschendes Lehren und forschendes Lernen - unter den andrängenden Massen junger Menschen ausgehöhlt, die Universitäten verkamen zu höheren Verwahranstalten; ${ }^{2}$ allein die For-

\footnotetext{
1 Vgl. Labisch (2004). Ich danke Hans Süssmuth für die Anregung, zum Abschluss meines Amtes als Rektor der HHU D eine - allerdings keineswegs rückwärts sondern vorwärts gewandte - Summe meiner Arbeit und meiner Erfahrungen zu ziehen.

2 Vgl. hierzu übrigens am Beispiel der Universität Düsseldorf den „Erziehungsroman“ zeitgenössischer Studenten: Heinzen und Koch (1989).
} 
schung hielt einigermaßen ihr Niveau. Festzuhalten ist: Auch heutzutage setzen alle finanziellen Versprechungen auf demjenigen niedrigen Niveau ein, das mit der permanenten Unterfinanzierung der Universitäten vorgegeben ist. Anders ausgedrückt: Von der Ausstattung der Zeit vor 1977, etwa in der Relation von Studierenden zu Lehrenden, sind wir heute weit entfernt.

Gleichwohl: Autonomie und Wettbewerb sind die hochschulpolitischen Schlüsselworte unserer Zeit. Am weitesten durchgesetzt wurde der Gedanke der Autonomie sicherlich in Nordrhein-Westfalen, dem Bundesland, in dem die Universitäten durch das so genannte Hochschulfreiheitsgesetz ihren Staatscharakter verloren haben und nunmehr weitgehend selbständige Personenkörperschaften sind. Demzufolge haben sich die Universitäten auch weitgehend selbständig im nationalen und internationalen Wettbewerb zu behaupten. Hat, so ist hier zu fragen, die Kultusbürokratie eingesehen, dass Universitäten zu komplex sind, um sie von außen steuern zu können? Sollte die sinkende Kapitalkraft öffentlicher Haushalte etwa der eigentliche Vater dieser Entscheidung sein? Oder spielen sich hinter den aktuellen Reformen möglicherweise tiefer greifende Prozesse ab?

\section{Universitäten als Teil gesellschaftlicher Produktion}

Dass in der Hochschulpolitik Einsicht gegeben ist, mag durchaus zutreffen. Allerdings spielt sich der Prozess, der hier und heute in Deutschland zu beobachten ist, weltweit ab. Wie wäre es sonst zu erklären, dass ein methodisch völlig unzureichendes Ranking der weltbesten Universitäten wie das der Jiao-Tong-Universität Shanghai der Jahre 2004ff. nicht nur in Deutschland, sondern weltweit eine solche öffentliche Aufmerksamkeit und nachfolgend einen solchen hochschulpolitischen Schub auslösen konnte? Spüren wir hier lediglich die Ausläufer einer weltweiten Welle?

Um die aktuellen Vorgänge besser zu verstehen, ist die zeitliche Perspektive und damit der Blick in die Geschichte hilfreich. Traditionell bilden Universitäten die künftigen Führungskräfte aus - dies war der Anlass, in Bischofskirchen und Handelsstädten höhere Lehranstalten zu schaffen. Allmählich wandelten sich die Universitäten, vorangetrieben durch Glaubensfragen innerhalb der una sancta catholica, zu Orten des freien, zumindest des anderen Denkens und - verschärft dann ab dem 19. Jahrhundert - in Stätten der Forschung. Der Wandel zu Forschungsuniversitäten speiste sich vornehmlich aus einer eigenen Dynamik.

Heute geschieht etwas völlig Neuartiges. Die Universitäten treten weltweit aus dem Schatten der gesellschaftlichen Reproduktion heraus. Es geht nicht mehr darum, gleichsam aus der zweiten Linie den Nachwuchs und die Expertise für jene gesellschaftliche Bereiche der ersten Linie zu liefern, in denen die wirtschaftlichen und sozialen Güter gehobenen Niveaus geschaffen werden. Unter dem Signum der Innovation als einer entscheidenden Ressource im globalen Wettbewerb wechseln die Universitäten vom konsumtiven Bereich der Reproduktion in den wertschaffenden Bereich der gesellschaftlichen Produktion. Führungskräfte gewiss - aber nicht mehr fünf Prozent sozialer Eliten, sondern 35, vielleicht sogar 50 Prozent wissenschaftlich ausgebildeter Mitarbeiter, wie sie die globale wissenschaftsbasierte Lebenswelt einfordert. Und neue wissenschaftliche Ideen stellen gleichsam den sich ständig neu schöpfenden Rohstoff dar, um mit wirtschaftlich verwertbaren Innovationen vor allen anderen auf einem globalen Markt bestehen zu können. 
Dieser Vorgang könnte durchaus plakativ mit dem Schlagwort „Globalisierung von universitärer Ausbildung, Wissenschaft und Technologie“ beschrieben werden. Was bedeutet dieser Vorgang für die Universitäten? Was gewinnen sie, was verlieren sie? Und was müssen die Universitäten unter allen Umständen bewahren, um sich in diesem weltweiten Wettbewerb von Talenten und Ideen als Universität behaupten zu können?

\section{Konsequenzen der Globalisierung von universitärer Ausbildung, Wissenschaft und Technologie}

Zunächst einmal ist unmittelbar einsichtig, dass sich das Beziehungsgefüge der Universitäten dramatisch ändert. Ein solches Beziehungsgefüge erklärt sich aus den Auftraggebern und Abnehmern der Produkte einer Universität. Eine Kathedralschule und spätere bischöfliche Universität stand in einem ebensolchen Bezugsverhältnis zur Kirche wie eine städtische Universität zu den führenden Schichten einer Stadt: Theologie, kirchliches Recht, in den Städten auch weltliches Recht und Medizin, das wurde gewünscht und auch gefördert, anderes eher geduldet oder gar bekämpft. Vergleichbares galt für die staatlichen Universitäten der Neuzeit: Theologie, Jus, Medizin - später dann auch wirtschaftliche, naturwissenschaftliche und technische Expertise. Die Universitäten auch wirtschaftlich frei zu stellen, in Preußen etwa durch die Mittel der staatlichen Domänen, wie dies Wilhelm von Humboldt ursprünglich vorschlug, wurde allerdings nicht erreicht. Seit dem frühen 19. Jahrhundert sind die Universitäten finanziell durchweg vom Staat abhängig. Seit dem Ende des Zweiten Weltkrieges und dem grundgesetzlichen Privileg der Freiheit für Wissenschaft und Forschung wird die Abhängigkeit vom Staat im universitären Alltag üblicherweise sogar so gedeutet, als ob die Universitäten frei seien. Große Stiftungen, konfessionelle oder private Universitäten stellen zumindest bis heute die Ausnahme von der Regel dar.

Tritt heute an die Stelle von Kirche oder Staat also die Wirtschaft? - so wäre leicht zu schlussfolgern. Dies wäre zu einfach. Die Universitäten treten auf einem globalen Markt von qualifiziertem Personal, Expertise, Forschung und forschungsgetriebener Innovation an. Das Beziehungsgefüge der Universitäten als selbständige und damit de facto unternehmerische Akteure gesellschaftlicher Produktion wird so tatsächlich auf die Teilhabe an einem globalen Markt erweitert, dessen konkurrierende Akteure unterschiedlich verfasste Unternehmen und unterschiedlich Körperschaften bis hin zu Staaten als Abnehmer und selbstverständlich alle anderen Universitäten als Anbieter sind. Auf diesem Markt gewinnen die Universitäten gleichsam ihre Rohstoffe und Produktionsmittel - von jungen Talenten bis hin zu weltweit renommieren Wissenschaftlern. Und hier finden die Universitäten auch die Abnehmer ihrer Leistungen: Wissenschaftliche Qualifikation, und zwar in Massen und über die Weiterbildung über die gesamte Erwerbsbiografie verteilt, Expertise und forschungsgetriebene Innovation sind die Produkte, die sich auf globalen Märkten behaupten müssen, um in die globalen Lebenswelten einzugehen.

Wir erleben also gleichzeitig zwei Prozesse, die vielleicht nur Erscheinungsformen eines einzigen grundlegenden Prozesses sind. Erstens gehen die Universitäten von der Reproduktion in die Produktion über. Dies geschieht zweitens zugleich unter globalen Vorgaben. Und beides ist wahrscheinlich nur ein Aspekt des Prozesses, in dem sich die Industriewelt derzeit als vorherrschende Lebensform auf die gesamte Erde ausweitet. Das neue Beziehungsgeflecht der Universitäten ist folglich ungleich komplexer als die zuvor 
gegebenen. Nicht mehr ein primärer Ansprechpartner - das zuständige Ministerium - sondern völlig neue Anforderungen durch neue Aufmerksamkeiten und neue Formen des Handelns, die sich gleichsam aus der Kette universitärer Produktion ergeben. Vom einzubringenden „Material“ - der Auswahl der Studierenden und Graduierenden - über die „Produktionsmittel“ - die besten Köpfe und die besten Forschungsmittel - bis hin zum „Vertrieb“ - also der Vermittlung der Absolventen und Graduierten, der Expertise und der Forschungsergebnisse auf einen globalen Markt. Zu dieser unmittelbaren Produktion tritt damit als notwendiges Medium die Kommunikation über sämtliche Bereiche des Produktionsprozesses - und zwar nach innen wie nach außen - bis hin zum Marketing. Internationalisierung beschreibt - zumindest in der geläufigen Interpretation akademischer Auslandsarbeit - nur unzulänglich, in welchen räumlichen Dimensionen die Universitäten jetzt denken müssen. ${ }^{3}$ Es wird in diesem Gesamtzusammenhang - der in der Tat mit einigen erhellenden Ideen in der Analogie zur Volks- und Betriebswirtschaft durchdacht werden sollte - eine wesentlich neue Aufgabe geben: nämlich den Transfer der Absolventen, des Expertenwissens und der Forschungsergebnisse auf einen Markt globaler Nachfrage.

\section{Konsequenzen eines globalen Prozesses für die deutschen Universitäten}

Was bedeutet dies nun für die deutschen Universitäten? Der Staat zieht sich aus der Steuerung der Universitäten zurück. Die Vorgaben, nach denen sich die Universitäten ausrichten, folgen aus einem hier angedeuteten globalen Wettbewerb sowohl im Bereich der Wissenschaften - was dem seit jeher internationalen Wissenschaftsbetrieb vertraut ist - als auch im Bereich eines akademischen Marktes - Absolventen, Expertise, Innovation. Wegen der steten Kostensteigerung verschlimmert sich die seit 30 Jahren - eben seit dem oben angesprochenen Öffnungsbeschluss von 1977 - bestehende Unterfinanzierung der Universitäten weiter. Der Anteil der fixen Finanzierung durch die öffentliche Hand fällt, der Teil an Finanzmitteln, der kompetitiv sowohl in der Lehre - durch Studienbeiträge - in der Forschung - kompetitive Drittmittel - als auch durch Transfer erworben werden muss, steigt. Ein immer größerer Anteil der Einnahmen einer Universität wird demnach aus der Grundfinanzierung in den kompetitiven Bereich verlagert - ebenfalls ein weltweites Phänomen, das sich besonders gut in den USA studieren lässt, in denen auch staatliche Universitäten inzwischen kaum mehr als 40 Prozent ihre Einnahmen aus staatlichen Mitteln gewinnen. Die Universität, die auf diese Entwicklung nicht reagiert, fällt folglich gegenüber kompetitiven Universitäten immer weiter zurück.

In Deutschland wird die internationale Wettbewerbsfähigkeit seit 2004 durch den so genannten Exzellenzwettbewerb hochschulpolitisch bewusst forciert. Den Universitäten wird der Schleier einer angeblich aus gleichen „Volluniversitäten“ bestehenden Universitätslandschaft abrupt weggezogen und damit gezielt jede Behäbigkeit ausgetrieben. Welche deutschen Universitäten sind international sichtbar? Welche gehören darüber hinaus zu dem kleinen Kreis weltweit anerkannter „Eliteuniversitäten“? Das sind die Kernfragen des so genannten ,Exzellenzwettbewerbes“. Der internationale und nationale Gradmesser der Differenzierung wird - trotz aller gegenteiliger Beteuerungen - zunächst die Forschung und nicht die Lehre sein. Forschung ist daher auch in Deutschland der Gradmesser. Er teilt

\footnotetext{
3 Vgl. Borsò (2005).
} 
die international sichtbaren Forschungsuniversitäten Deutschlands - es werden dies etwa 25 Prozent sein - von den primären Lehruniversitäten. Vergleichbar ist hierzu das Verhältnis von Forschungsuniversitäten (circa 100) zu den weiteren höheren Ausbildungsstätten (circa 4.000) in den USA. Dabei stehen Forschung und Lehre in einem Wechselverhältnis. Nur diejenige Universität, die in der Lage ist, die talentiertesten Studierenden anzuziehen, wird in der Forschung genügend Nachwuchs haben. Allerdings: Diejenige Universität, die in der Forschung exzellent ist, zieht eben diese Talente auch international an. Dies ist beispielsweise an den deutschen Einrichtungen zu sehen, die von Nobelpreisträgern geführt werden. Es gibt folglich nicht nur einen globalen Wettbewerb in der Forschung, sondern auch in der Lehre. Und eben deshalb kann die Universität, die zwar eine gute Forschung, aber keine gute Lehre betreibt, keine Eliteuniversität sein. Allerdings: Ohne herausragende Forschung wird es auch keine herausragende Lehre geben. Lehre und Forschung bedingen einander, aber gute Lehre folgt stets der guten Forschung.

Im Ergebnis wird sich die deutsche Universitätslandschaft in einige wenige weltweit anerkannte Exzellenzuniversitäten, in wenige international agierende Forschungsuniversitäten und in viele teils nationale, teils regionale Lehruniversitäten differenzieren, die die Masse wissenschaftlich qualifizierter Fachkräfte ausbilden werden. Aus der ersten Runde der Exzellenzinitiative ist bekannt, dass in diesem Wettbewerb nur diejenigen Universitäten eine Chance haben, die über eine hinreichende Größe verfügen. ${ }^{4}$ Es sind dies rein empirisch mindestens 400 Lehrstühle und mehr. Oder aber es handelt sich um so genannte Spartenuniversitäten, in denen sich eine Vielzahl von Lehrstühlen oder Instituten um ein großes Thema schart - also beispielsweise Technische Universitäten. Überlebenschancen als Forschungsuniversitäten haben auch unter den großen Universitäten nur diejenigen, in deren Umgebung eine Vielzahl herausragender Forschungsinstitutionen für eine unmittelbare Kooperation vorhanden sind - Institute der an sich bereits weltweit beispielhaften Max-Planck-Gesellschaft, Großforschungseinrichtungen der Helmholtz-Gesellschaft, die vielfältig ausgerichteten Institute der Wissenschaftsgemeinschaft Leibniz und gegebenenfalls auch Institute der angewandten und industrienahen Fraunhofer-Gesellschaft. Dort, wo alles dies nicht in ausreichendem Maße oder gar überhaupt nicht gegeben ist, bleibt für eine international sichtbare Konkurrenz nur eines: konsequente und über viele Jahre ausgebaute Konzentration auf einen oder zwei Forschungsbereiche, die das Potenzial zu weltweiter Sichtbarkeit haben. Eines der wenigen, vielleicht sogar das einzige Beispiel dafür ist die Universität Konstanz. Und wo das alles nicht gegeben ist, bleibt eben nur der Weg in die Zweitklassigkeit.

\section{Konsequenzen einer weltweiten Entwicklung für die Heinrich-Heine-Universität}

Was diese Entwicklung für die Heinrich-Heine-Universität Düsseldorf bedeutet, ist an sich aus dem vorher Gesagten Punkt für Punkt abzuleiten - sofern zuvor die zeitlichen und örtlichen Gegebenheiten der Heinrich-Heine-Universität dargelegt und beachtet werden. Die Heinrich-Heine-Universität ist eine junge und eine kleine Universität. Die Heinrich-HeineUniversität leidet nach wie vor an Strukturdefiziten, die aus der ,schleichenden Universi-

\footnotetext{
${ }^{4}$ Vgl. hierzu die bemerkenswerte Analyse von Leibfried und Wiesner (2008).
} 
tätsgründung “5 folgen. Für diese Universität, die als bislang letzte Hochschule des Landes Nordrhein-Westfalen als Universität - und nicht als Gesamthochschule - gegründet wurde, hat es im Gegensatz zu den anderen Universitätsgründungen der 1960er Jahre (Bielefeld, Bochum, Dortmund) weder ein hochschulpolitisches Gesamtkonzept noch die entsprechenden Ausbau- und Ressourcenpläne gegeben. Damit folgen Strukturdefizite auch aus der ,,unterkritischen Größe“ der Universität. Die Heinrich-Heine-Universität hat insgesamt wenig mehr als 300 Professoren. Schließlich folgen wesentliche Strukturdefizite aus der Tatsache, dass es nur wenige geeignete und hinreichend große extrauniversitäre wissenschaftliche Kooperationspartner unmittelbar vor Ort gibt.

Trotzdem ist die Heinrich-Heine-Universität aufgrund ihrer steten und international sichtbaren Forschungsleistungen in Sonderheit in der Medizin, in den Lebens- und in den Naturwissenschaften eine „Schwellenuniversität“, eine „Universität auf dem Sprung“: Die Heinrich-Heine-Universität hat das Potenzial, sich auf Dauer unter den Forschungsuniversitäten Deutschlands zu etablieren. Grundvoraussetzung dafür ist die internationale Exzellenz in der Forschung. Daraus resultiert - wie oben dargelegt - zugleich die Aufgabe, die Lehre und die Graduiertenausbildung auf internationales Niveau zu bringen. Diese Ziele zu erreichen ist für das Überleben der Universität als anerkannter Stätte des Forschens und Lehrens elementar. Oder um es im Jargon auszudrücken: Für die Champions-League wird es nie reichen, aber ein fester Platz in der Bundesliga sollte es schon sein.

Überdies hat die Heinrich-Heine-Universität einen Standortvorteil, den keine nordrheinwestfälische Universität und den nur wenige deutsche Universitäten aufweisen können. Damit ist nicht der Umstand gemeint, dass die Universität in der Landeshauptstadt liegt. Dies hat der Universität - wie auch der Vergleich mit anderen Universitäten von Landeshauptstädten leicht ergibt - im Flächenland Nordrhein-Westfalen mit seiner traditionellen Standortpolitik nach Proporz und Wählerstimmen bislang nur wenig genutzt. Vielmehr liegt die Heinrich-Heine-Universität in einer der Zukunftsregionen Deutschlands. Düsseldorf selbst gilt als „Zukunftsstadt“. Überdies gilt der Großraum Düsseldorf als „Zukunftsregion“. Die allgemeine Aufgabe für eine Zukunftsregion lautet mit Blick auf eine Universität, Grundlagenforschung und angewandte wissenschaftliche Dienstleistungen, Produktion und Logistik geografisch zu konzentrieren.

In einer globalen, wissenschaftsbasierten Welt sind Universitäten ein entscheidendes Produktionsmittel einer Zukunftsstadt in einer Zukunftsregion. Eine wirtschaftlich prosperierende, und das heißt heute immer auch eine global agierende Region, ist auf eine leistungsfähige Universität angewiesen. Ebenso hat die Universität den Vorteil, in einem wirtschaftlich prosperierenden Gebiet zu liegen. Sowohl die Heinrich-Heine-Universität als auch die Zukunftsregion Düsseldorf bieten sich demzufolge gegenseitig Standortvorteile. Den Transfer zwischen Universität und den Gebietskörperschaften, Verbänden, Unternehmen und den Bürgern in Stadt und Region auszubauen ist also für die HeinrichHeine-Universität eine essenzielle Aufgabe.

Unter der Maßgabe allgemeiner Vorgaben und der zeitlich-örtlichen Besonderheiten hat eine Universität in der Größenordnung wie die Heinrich-Heine-Universität damit folgende allgemeine Aufgaben zu bewältigen:

5 Zum durchaus zutreffenden Begriff „schleichende Universitätsgründung“ siehe Plassmann und Riener (2003). 
Aus ihrer disziplinären Vielfalt heraus muss sich die Universität auf wenige Forschungsschwerpunkte konzentrieren. Über Fächer und Fakultäten hinweg müssen sämtliche möglichen Ressourcen in diese Schwerpunkte eingebracht werden, sofern sie den internationalen Forschungsstandards genügen. Interne Kooperation und Interdisziplinarität sind also das Gebot der Stunde.

Aus diesen Forschungsschwerpunkten kann die Universität wegen der notwendigen Ressourcen in wenigen, wohl höchstens fünf Gebieten eine nationale Mitsprache anstreben. Aus demselben Grund können dies auf internationalem Niveau höchstens zwei Themenbereiche sein. Eine Themenführerschaft auf nationaler oder gar internationaler Ebene würde den Konzentrationsprozess zu Lasten notwendiger universitärer Vielfalt zu weit führen.

Analog zur Forschung muss die Universität in wenigen ausgewählten Bereichen die nationale, gegebenenfalls auch internationale Exzellenz in der Lehre, in der Graduiertenausbildung und in der Weiterbildung erreichen. Es werden dies zwar vornehmlich, aber nicht ausschließlich diejenigen Gebiete sein, in denen auch die wissenschaftliche und forscherische Exzellenz gegeben ist.

Nur in enger Kooperation mit ihrerseits international exzellent agierenden außeruniversitären Forschungseinrichtungen kann die Universität selbst Exzellenz erreichen. Die inneruniversitäre Kooperation muss folglich durch die extrauniversitäre Kooperation ergänzt werden.

Die Basis für ihre nationalen und internationalen Aktivitäten ist der Transfer mit den Körperschaften und Unternehmen der Region und darüber hinaus. Dies gilt insbesondere auch für diejenigen Fakultäten, die durch ihre Expertise in entsprechende kulturelle, gesellschaftliche, politische, juristische oder wirtschaftliche Produktionsprozesse eingebunden sind.

Die unabdingbare Grundlage der Aktivitäten in Forschung und Lehre sind demnach Kommunikation und Transfer auf nationaler und internationaler Ebene.

\section{Gefahrenmomente für eine unternehmerische Universität}

Die vorgenannten Aufgaben ergeben sich aus der Entwicklung, wie sie sich derzeit für die Universitäten darstellt. Gleichwohl stellen sie die Vorgaben für eine weitreichende Strategie dar, die sich nur über längere Zeiträume durchsetzen lässt. Diese Strategie muss nicht notwendig zum Erfolg führen. Denn alle Universitäten, die sich eine Zukunftsperspektive sichern wollen, entwickeln selbstverständlich ähnliche Strategien, handeln entsprechend oder können von Zufällen profitieren. Ein Beispiel ist die Einrichtung des Max-PlanckInstituts für die Biologie des Alterns in Köln, das einem durchaus möglichen Exzellenzcluster Alternsforschung an der Heinrich-Heine-Universität den Boden entzogen hat. Die Universitäten sind in einen wirklichen und ernsthaften Wettbewerb eingetreten, in dem es notwendig auch Verlierer geben wird.

Das Absinken zur Lehruniversität ist die eine Gefahr. Die andere Gefahr resultiert daraus, im Konkurrenzkampf eines globalen Bildungs- und Innovationsmarktes die Wesensmerkmale universitären Forschens und Lehrens in einer Weise zu vernachlässigen, dass dadurch Forschung und Lehre gefährdet werden. Diese Gefahrenmomente müssen daher diskutiert werden. 


\section{Autonomie als Wesenszug universitären Forschens, Lehrens und Lernens}

Die Autonomie einer Universität ist kein Selbstzweck. Vielmehr ist Autonomie ein Mittel - aber eben das entscheidende, die conditio sine qua non: Die Universität beansprucht Autonomie, weil sie für sich das Recht und die Pflicht einfordert, alles zu erforschen, alles zu lehren, alles zu lernen, was im Interesse eines auf Erkenntnis gerichteten Fragens, Forschens und Wissens auch immer zu tun ist. Erst damit können die Universitäten die eigentlichen Stätten, gegebenenfalls sogar die Motoren neuen Denkens sein.

Hier liegt das wissenschaftstheoretische Moment, Autonomie auch wissenschaftspolitisch einzufordern. Die „Kern“-Kompetenz einer Universität gehorcht einer besonderen Ratio: Eine Universität ist ein Möglichkeitsraum für Ideen und für Talente. Talente entwickeln sich nur im Freiraum exzellenter Lehre. Daher stammt das Ziel, dass sich Lehrer und Studierende in der unmittelbaren Forschungssituation begegnen müssen. Historisch gesehen war dies der Schlüssel für die Wissensexplosion in den deutschen Universitäten der zweiten Hälfte des 19. Jahrhunderts. Ideen entwickeln sich nur im Freiraum zweckfreier Forschung. Neue Ideen in der Forschung sind kontingent - das heißt, sie sind einer bestimmten Zufälligkeit unterworfen. Ebenso ist es dieser bestimmten, weil bewusst herbeigeführten Zufälligkeit zu verdanken, dass sich neue Talente in der Lehre, besonders im lernenden Forschen zeigen und auch beweisen. Beides - neue Ideen und neue Talente sind also wesentlich selbst gesteuerte Prozesse. Wilhelm von Humboldt, der Propagator und gleichzeitig Gründer der modernen Universität, schreibt: ${ }^{6}$

„Der Universität ist vorbehalten, was nur der Mensch durch und in sich selbst finden kann, die Einsicht in die reine Wissenschaft. Zu diesem SelbstActus im eigentlichen Verstand ist notwendig Freiheit und hilfreich Einsamkeit, und aus diesen beiden Punkten fließt zugleich die ganze äußere Organisation der Universitäten. Das Kollegienhören ist Nebensache, das wesentliche, daß man in enger Gemeinschaft mit Gleichgesinnten und Gleichaltrigen und dem Bewußtsein, daß es am gleichen Ort eine Zahl schon vollendet Gebildeter gebe, die sich nur der Erhöhung und Verbreitung der Wissenschaft widmen, eine Reihe von Jahren sich und der Wissenschaft lebe.“

Als wesenhaft ,zufällig“ müssen sich neue Ideen und neue Talente folglich von selbst entwickeln können. Autonomie und das Ermöglichen von autonomen Lehr- und Forschungsprozessen sind damit wesentliche Bedingungen, eine Universität überhaupt produktiv in den nach außen gesetzten Transferzielen wirken zu lassen. Die Universität muss immer ein Platz auch für Personen und Fächer sein, denen externe Kriterien nicht gerecht werden und/oder die aufgrund ihrer exzellenten Arbeit externe Kriterien nicht benötigen. Anders ausgedrückt: Der einsame Denker, der einsame Experimentator klassischer Prägung muss seinen Platz an der Universität haben.

\footnotetext{
6 Wilhelm von Humboldt (1964: 191); vgl. hierzu den bereits oben zitierten Aufsatz „Die ,Idee der Universität in unserer Zeit“ (Labisch 2004) und Labisch (2005).
} 


\section{Autonomie auf Dauer: Die Heinrich-Heine-Universität als Stiftungsuniversität}

Unter den Bedingungen des Hochschulfreiheitsgesetzes kann die Heinrich-Heine-Universität autonom handeln. Wie die jüngsten hochschulpolitischen Diskussionen etwa zur Novelle des Hochschulfreiheitsgesetzes oder zur Abschaffung der Studienbeiträge zeigt, ist die neue gewonnene Autonomie bereits gefährdet. Autonomie ist eine der wesentlichen Grundlagen für die zukünftige Position der Universität. Autonomie ist daher auf Dauer oder - mit einem Modewort der Zeit - nachhaltig zu sichern. Deshalb arbeitet die Heinrich-Heine-Universität bereits seit vielen Jahren auf den Status einer Stiftungsuniversität hin.

Beispielhaft unter den bislang in Deutschland möglichen Varianten könnte eine öffentlich-rechtliche Stiftung nach dem Dresdner Modell sein. ${ }^{7}$ Diese Form einer Universitätsstiftung würde der Heinrich-Heine-Universität eine Reihe von Vorteilen bieten: Die Universität wäre weniger von politischen Tages- oder Wahlentscheidungen abhängig. Es wäre eine engere Kooperation zwischen der Universität, der Medizinischen Fakultät und dem Universitätsklinikum möglich: Das Universitätsklinikum und die Medizinischen Fakultät wären gleichsam eine „Unter“-Stiftung in der Universitätsstiftung. Es gäbe einen stärkeren Bestandsschutz. Die Gründungsvoraussetzung wäre, dass die Liegenschaften im Besitz der Universität wären. Eine weitere Voraussetzung wären Matching-Funds mit dem Land. Dies bedeutet, dass durch die Universität eingeworbene Stiftungen durch das Land entsprechend ergänzt werden. Dies setzt wiederum ein gehöriges Einkommen durch eigene Stiftungen voraus. Als Stiftungsuniversität wäre schließlich eine bessere Wahrnehmung und dadurch bessere Einbindung in die Region möglich. In den beiden letztgenannten Punkten käme ein weiteres Moment aus der besonderen Lage der Heinrich-Heine-Universität zum Tragen: die Stiftermentalität der Bürgerinnen und Bürger in der Region Düsseldorf, die sich um ,ihre“ Universität tatkräftig sorgen.

\section{Bildung für die Lehrenden und Lernenden: Zur notwendigen Integration von Fachwissen, Allgemeinbildung und Persönlichkeitsbildung}

Die Zukunft wird eine globale industrielle Lebenswelt mit globalen Möglichkeiten und Herausforderungen sein. Um in einer solchen Welt nicht nur etwas zu leisten, sondern die Zukunft mit zu gestalten, ist mehr erforderlich als Fachwissen und Expertise. Die Zukunft wird nicht von Bürokraten oder Technokraten, sondern von Menschen gestaltet, die in einem breiten Horizont von Wissen und Erfahrung Visionen für das zukünftig Nötige und Mögliche entwickeln und diese Visionen charakterstark und umsichtig in die Tat umsetzen können. Dazu nur ein Beispiel globaler universitärer Wirklichkeit: Chinesische Universitäten, allen voran die Chinese University of Hongkong, führen neuerdings für alle Studierenden verpflichtend humanistic studies ein. Wichtig ist also nicht nur die Autonomie der Universität, sondern auch ein wesentliches, allerdings zumeist verborgenes Merkmal

\footnotetext{
7 Vgl. Post (2005); vgl. hierzu demnächst auch „Die Stiftungsuniversität - ein Modell für die Heinrich-HeineUniversität? Symposium anlässlich des 60sten Geburtstages des Kanzlers der Heinrich-Heine-Universität, Herrn Prof. Ulf Pallme König, Düsseldorf 2008 (Schriftenreihe Mosaik der Heinrich-Heine-Universität).
} 
universitären Denkens und Handelns: Bildung - und zwar nicht als Kanon angeblich zu wissenden Wissens, sondern als innere Haltung. Nicht nur Ausbildung, sondern Bildung tut also Not. Bildung im Sinne von Haltung ist - so in der Nachfolge Georg Friedrich Wilhelm Hegels - im berühmten delphischen Gebot des „Erkenne dich selbst“" zu fassen. ${ }^{8}$

Diese eher grundsätzlichen Ausführungen, warum Bildung in den Kern einer Universität gehört, bekommen in Deutschland durch den Druck äußerer hochschulpolitischer Ereignisse zusätzlich Schub. Der Bologna-Prozess läuft nicht nur unter dem Aspekt der Internationalisierung der Studienabschlüsse, sondern auch unter dem Aspekt der Berufsorientierung. Dies führt dazu, dass zumindest für die Phase des Bachelorstudiums akademisches Lehren und Lernen eingeschränkt sind. Hier setzt jener bereits zuvor geäußerte Gedanke an, über die generelle Idee des Bildungsgedankens der Universität hinaus die Frage zu stellen, ob das berufliche Fach-Wissen, die berufliche Fach-Aus-Bildung in einer Zeit genügen, die sich unter dem Signum der Globalisierung neu ausrichtet? Ferner ist mit der in Deutschland möglicherweise allzu strikt - durchgeführten Studienreform ein weltweit anerkanntes Charakteristikum deutscher Hochschulabsolventen bedroht: die Fähigkeit zur selbständigen geistigen Arbeit. Hier stellt sich die Frage, ob es in der Universität weniger darum geht, ein vorgegebenes Pensum als vielmehr selbständiges Denken und Forschen zu lernen.

Angesichts dieser teils theoretisch, teils praktisch begründeten Erfordernisse muss die Heinrich-Heine-Universität auf umfassende Bildung setzen. Leben, Werk und Vermächtnis ihres Namenspatrons Heinrich Heine können der Universität als dauernder Ansporn dienen. „Brillanter Intellekt, weltoffener Geist, ein weites Herz“ - dieses Wort ist mittlerweile als Markenzeichen der Heinrich-Heine-Universität rechtlich geschützt. Unter dieser Vorgabe wurde seit 2005 mit dem Aufbau eines „Studium Universale“ begonnen. Mittlerweile in den meisten Bachelorstudiengängen Pflicht, geht es im Studium Universale ebenso um allgemeine Bildung wie um Schlüsselqualifikationen. Seit dem 1. Juli 2008 ist das Zentrum für das Studium Universale eine Zentrale Wissenschaftliche Einrichtung der Heinrich-Heine-Universität. ${ }^{9}$ Des Weiteren verfolgt die Heinrich-Heine-Universität das ConceptCampusCultur: Musik, Sport, Theater oder der Kunstpfad ${ }^{10}$ seien genannt. Bildung kann gelingen, wenn universitäres Lehren und Lernen in der persönlicher Begegnung mit anderen erlebt wird. Das Studium ist eine Phase des Lebens, die Halt für das künftige Leben gibt. Mit diesen Vorgaben könnte auf dem einzigartigen Campus der Heinrich-HeineUniversität - eine Universität und ein Universitätsklinikum auf einem Areal - ein eigenes kulturelles Milieu entstehen, das „Bildung“ im weitesten Sinne nicht nur ermöglicht, sondern als selbstverständlichen Teil universitären Lebens geschehen lässt.

\section{Die Idee der Universität in unserer Zeit - Ein Fazit}

Die globale Welt setzt auf Produktion durch Innovation. Damit werden die Universitäten zu Stätten primärer gesellschaftlicher Wertschöpfung. Und zugleich werden damit die Universitäten als Produzenten von Forschungsergebnissen, Expertise und Absolventen Akteure in einem für sie neuen Bezugssystem: An die Stelle von Kirche oder Staat

\footnotetext{
8 Vgl. hierzu Siep (2003) und Labisch (2004).

9 Vgl. auf der Horst (2006).

${ }^{10}$ Vgl. hierzu auch als Ergebnis eines Projektes des Seminars für Kunstgeschichte: Beckers et al. (2006).
} 
treten Körperschaften und Unternehmen einerseits sowie konkurrierenden Universitäten andererseits. Die Universitäten müssen sich in neuen Beziehungsgeflechten durch den Transfer ihrer „Produkte“ im Wettbewerb behaupten. Dies setzt eine völlig andere Qualität der Kommunikation voraus. Internationalisierung heißt jetzt, in den Bezügen eines global campus zu denken und zu handeln.

Was dies für die Heinrich-Heine-Universität bedeutet, lässt sich leicht ableiten. Trotz der Defizite, die durch die ,schleichende Universitätsgründung“ bedingt sind, und trotz ihrer unterkritischen Größe und ihrer Lage fern von außeruniversitären Forschungseinrichtungen ist die Heinrich-Heine-Universität aufgrund ihrer Forschungsleistungen durchaus in der Lage, sich auf Dauer in der Liga der deutschen Forschungsuniversitäten zu etablieren. Um dies zu erreichen, muss sich die Heinrich-Heine-Universität auf wenige übergreifende Forschungsschwerpunkte konzentrieren. Aus diesen Forschungsschwerpunkten kann die Heinrich-Heine-Universität in wenigen ausgewählten Gebieten eine nationale Mitsprache anstreben. Auf internationalem Niveau können dies bis zu zwei Themenbereiche sein. Analog zur Forschung muss die Heinrich-Heine-Universität in der akademischen Lehre, der Graduiertenausbildung und auch in der Weiterbildung in wenigen Bereichen national, gegebenenfalls auch international exzellent sein. Dies kann die Heinrich-Heine-Universität nur in enger Kooperation mit ihrerseits international exzellent agierenden außeruniversitären Forschungseinrichtungen erreichen. Die Basis für ihre nationalen und internationalen Aktivitäten ist der Transfer mit den Körperschaften und Unternehmen der Region und darüber hinaus. Die unabdingbare Grundlage dieser Aktivitäten in Forschung und Lehre sind Kommunikation und Transfer auf nationaler und internationaler Ebene.

In dieser neuen Welt der Universitäten sind zwei an sich seit alters her gegebene Merkmale wichtig: Autonomie und Bildung. Autonomie erklärt sich zwingend aus dem Produkt der Universitäten: Die „heiße Zelle“ einer Universität ist die Dyade von Lehrendem und Lernendem beziehungsweise Forscher und Studierendem in dem möglichkeitsoffenen Raum, denjenigen Fragen nachzugehen, die ihnen aus sich selbst heraus wichtig sind. Erst an der Grenzerfahrung des eigenen Könnens erweist sich das Talent. Und erst aus der Grenzerfahrung von bekanntem Wissen und eigener forscherischer Anstrengung folgen Innovation und Transfer. Um in diesem wettbewerblich bestimmten Bezugssystem die notwendige Autonomie zu bewahren, ist es geboten, die Universitäten über Stiftungen abzusichern, die in großen Teilen aus eigenem Kapital getragen werden. Hierfür hat die Heinrich-Heine-Universität hervorragende Voraussetzungen.

Wenn es der Kern einer autonomen Universität ist, Talente und Ideen zu entdecken, dann handelt es sich um Grenzerfahrungen, die uns auch zum Kern des Begriffes Bildung bringen. Die Begegnung mit sich selbst in der Grenzsituation eines auf neue Talente gerichteten Lehrens und Lernens sowie eines auf grundsätzlich Neues gerichteten Forschens ist auf das gerichtet, was als Bildung im Sinne des „Erkenne dich selbst“ verstanden werden kann. Bildung gehört damit aus sich heraus in den inneren Kern der Universität.

Die Herausforderung, die das Leben und Wirken in der Universität darstellt, findet sich mutatis mutandis - in wunderbar kurzer Weise in dem bekannten Satz Hugo von Hofmannsthals: „Der Mensch wird in der Welt nur das gewahr, was schon in ihm liegt, aber er braucht die Welt, um gewahr zu werden, was in ihm liegt; dazu sind Tätigkeit und Lei- 
den nötig" ${ }^{11}$ - und dies heißt für uns: in universitärer Autonomie forschen, lehren und lernen. Dies zu ermöglichen würde die Heinrich-Heine-Universität tatsächlich zur Elite aufschließen lassen.

\section{Literatur}

AUF DER HORST, Christoph (2006). „Das Studium Universale der Heinrich-Heine-Universität zwischen ,akademeia' und ,universitas “", in: Alfons LABISCH (Hrsg.). Jahrbuch der HeinrichHeine-Universität Düsseldorf 2005/2006. Düsseldorf 2006, 41-50.

Beckers, Anne, Barbara Frings, Ana SChepkowski und Nina TeUsch (2006). campus:construction. Heinrich-Heine-Universität. Vier Präsentationen des Projekts „Kunstpfad“ des Seminars für Kunstgeschichte zur Campus-Neugestaltung. Düsseldorf o.J.

Borsò, Vittoria (2005). „Internationalisierung als Aufgabe der Universität“, in: Alfons LABISCH (Hrsg.). Jahrbuch der Heinrich-Heine-Universität Düsseldorf 2004. Düsseldorf, 33-49.

HeInZEN, Georg und Uwe Koch (1989). Von der Nutzlosigkeit, erwachsen zu werden. Reinbek 1989.

Hofmannsthal, Hugo von (1922). Buch der Freunde. Tagebuch-Aufzeichnungen. Leipzig.

Humboldt, Wilhelm von (1964). „Unmaßgebliche Gedanken über den Plan zur Errichtung des Litauischen Schulwesens“, in: Werke in fünf Bänden, herausgegeben von Andreas FLITNER und Klaus GiEL. Darmstadt, Bd. 4, 187-195.

LABISCH, Alfons (2004). „Die ,Idee der Universität“ in unserer Zeit. Analysen und Konsequenzen“, in: Alfons LABISCH (Hrsg.). Jahrbuch der Heinrich-Heine-Universität Düsseldorf 2003. Düsseldorf, 23-38.

LABISCH, Alfons (2005). ,Autonomie der Universität - Ein Leitbild für die Heinrich-Heine-Universität Düsseldorf“, in: Alfons LABISCH (Hrsg.). Jahrbuch der Heinrich-Heine-Universität Düsseldorf 2004. Düsseldorf 2005, 19-32.

LEIBFRIED, Stephan und Achim WIESNER (2008). „Exzellenzinitiative: Wie soll es weitergehen?“, Frankfurter Allgemeine Zeitung (08.07.2008).

Plassmann, Max und Karoline Riener (2003). „Die ersten Jahre der Universität Düsseldorf (1965-1970) - Von der ,schleichenden“ Gründung bis zum Namensstreit“, in: Gert KAISER (Hrsg.). Jahrbuch der Heinrich-Heine-Universität Düsseldorf 2002. Düsseldorf 2003, 503-512.

Post, Alfred (Hrsg., 2005). Stiftungsuniversität Technische Universität Dresden. Zur Struktur von Universität und Hochschulmedizin in Dresden. Symposium am 16. März 2005 im Festsaal des Rektors der Technischen Universität Dresden, Dresden. (Dresdner Studien zur Bildungs- und Hochschulplanung; Bd. 6)

SIEP, Ludwig (2003). „Hegel und Europa“. Vortrag auf der Jahresfeier der Nordrhein-Westfälischen Akademie der Wissenschaften. Mittwoch, 7. Mai 2003. Düsseldorf.

\footnotetext{
${ }^{11}$ von Hofmannsthal (1922).
} 